\title{
FREE INVERSE SEMIGROUPS
}

Dedicated to the memory of Hanna Neumann

G. B. PRESTON

(Received 22 June 1972)

Communicated by M. F. Newman

In an important recent paper H. E. Scheiblich gave a construction of free inverse semigroups that throws considerable light on their structure [1]. In this note we give an alternative description of free inverse semigroups. What Scheiblich did was to construct a free inverse semigroup as a semigroup of isomorphisms between principal ideals of a semilattice $E$, say, thus realising free inverse semigroups as inverse subsemigroups of the semigroup $T_{E}$, a kind of inverse semigroup introduced and exploited by W. D. Munn [2]. We go instead directly to canonical forms for the elements of a free inverse semigroup. The connexion between our construction and that of Scheiblich's will be clear. There are several alternative procedures possible to reach our construction on which we comment on the way.

\section{Introduction}

Let $X$ be a non-empty set. Let $X^{-1}=\left\{x^{-1} \mid x \in X\right\}$ be a set disjoint from $X$, where $x \mapsto x^{-1}$ is a one-to-one mapping of $X$ upon $X^{-1}$. Set $Y=X \cup X^{-1}$. Denote by $\mathscr{F}_{Y}$ the free semigroup on $Y$ : the elements of $\mathscr{F}_{Y}$ are the non-empty words in the alphabet $Y$ and the product of any two words $u, v$ in $\mathscr{F}_{Y}$ is the word $u v$ obtained by juxtaposition of $u$ and $v \cdot \mathscr{F}_{Y}^{1}$ denotes the semigroup with identity obtained from $\mathscr{F}_{Y}$ by adjoining an identity element 1 to $\mathscr{F}_{Y}$. We shall also call 1 a word.

A word in $\mathscr{F}_{Y}$ is said to be reduced if it does not contain a syllable $x x^{-1}$ or a syllable $x^{-1} x, x \in X$, as a subword. The word 1 of $\mathscr{F}_{Y}^{1}$ is also said to be reduced. Any word of $\mathscr{F}_{Y}^{1}$ determines a unique reduced word obtained from it by deleting, in succession, any syllables $x x^{-1}$ or $x^{-1} x, x \in X$. The set of all reduced words in $\mathscr{F}_{Y}^{1}$ we shall denote by $G . G$ is then the free group on $X$ under the product

$$
::(u, v) \rightarrow u \cdot v, u, v \in G,
$$

where $u \cdot v$ denotes the reduced word determined by $u v$. 
If $g$ is an element of $G$ we shall denote its inverse in $G$ by $g^{-1}$. Set

$$
F=\left\{g_{1} g_{1}^{-1} \cdots g_{k} g_{k}^{-1} \mid k \geqq 1, \text { each } g_{i} \in G \backslash\{1\}\right\},
$$

and set $F^{1}=F \cup\{1\}$. Then $F$ is a subsemigroup of $\mathscr{F}_{Y}$ and $F^{1}$ is a subsemigroup of $\mathscr{F}_{Y}^{1}$. Denote by $W^{1}$ the set $F^{1} G$ :

$$
W^{1}=\left\{f g \mid f \in F^{1}, g \in G\right\} .
$$

The product $f g$ is here to be evaluated in $\mathscr{F}_{Y}^{1}$. Finally set $W=W^{1} \mid\{1\}$.

There are now several possible ways of proceeding to construct $I_{X}$, the free inverse semigroup on $X$. We shall proceed by first constructing a semilattice $E$, say, that will form the semilattice of idempotents of $I_{X}$. We shall then form a semi-direct product* of $E^{1}$ and $G$. The resulting semigroup $J_{X}^{1}$ is not an inverse semigroup and indeed is not regular. We shall then define a congruence on $J_{X}^{1}$ such that each congruence class contains precisely one regular element. The quotient of $J_{X}^{1}$ modulo this congruence will be $I_{X}^{1}$. Finally $I_{X}^{1}=I_{X} \backslash\{1\}$. Alternatively $I_{X}^{1}$ may be identified with the semigroup of regular elements of $J_{X}^{1}$; and this is perhaps the simpler approach.

It will be seen at the end that what has effectively been done is to introduce an equivalence relation on $W$ and then to define a product on the equivalence classes to give $I_{X}$. Although this latter procedure is on the face of it more direct - we shall give the formal definitions required later - the verification that the constructed object $I_{X}$ is an inverse semigroup involves much the same argument as in the procedure we have chosen to adopt.

Another possible procedure would be to consider the cartesian product $E^{1} \times G$, define an equivalence on this, and then define a product on the resulting set of equivalence classes to give $I_{X}^{1}$ and so $I_{X}$. We shall not give the details of this approach.

For each of the approaches mentioned we can instead go directly to $I_{X}$ and suppress the introduction of the 1 . However the introduction of the 1 smoothes the computational path.

\section{The construction of $I_{X}$}

LEMMA 1. Each element of $F$ is uniquely expressible in the form $g_{1} g_{1}^{-1}$ $\cdots g_{k} g_{k}^{-1}$, where $k \geqq 1$, and $g_{i} \in G \backslash\{1\}, i=1,2, \cdots, k$.

Proof. Let $a=g_{1} g_{1}^{-1} \cdots g_{k} g_{k}^{-1}$. Then, as an element of $\mathscr{F}_{Y}, a$ is a unique product of elements of $Y, a=y_{1} \cdots y_{n}$, say. Each $y_{j}$ is itself a reduced word, and so belongs to $G$. Consider the product in $G, y_{1} \cdot \ldots \cdot y_{n}$. As we successively evaluate $y_{1}, y_{1} \cdot y_{2}, \cdots$, it is clear that the first time we get the value 1 is when we have

* What we term a semi-direct product (see below) is a generalization of the usual concept. 
evaluated the product as far as the initial segment $g_{1} g_{1}^{-1}$ of $a$. This determines $g_{1}$ uniquely. Similarly, the next time the value of the product is 1 is on reaching the end of the initial segment $g_{1} g_{1}^{-1} g_{2} g_{2}^{-1}$. This determines $g_{2}$ uniquely. A continuation of the argument shows that the remaining $g_{j}$ are also uniquely determined.

Let us agree to write $g \leqq h$, for $g, h$ in $G$, if $g$ is an initial segment of $h$, i.e. if there exists $g^{\prime}$, say, in $G$, such that $g \cdot g^{\prime}=g g^{\prime}=h$. Here, to require that $g \cdot g^{\prime}$ $=g g^{\prime}$ means requiring either that $g g^{\prime}$ is reduced as it stands or that one of $g$ and $g^{\prime}$ equals 1 . Hence, for example, $1 \leqq g$, for all $g$ in $G$. Then $\leqq$ is a partial order on $G$.

If $A$ and $B$ are nonempty subsets of $G$, we mean by $A \leqq B$ that each element of $A$ is less than some element of $B$. If $A$ is finite then Max $A$ will denote the set of its maximal elements.

Because of Lemma 1 , if $f=g_{1} g_{1}^{-1} \cdots g_{k} g_{k}^{-1} \in F$, then the set $\left\{g_{1}, \cdots, g_{k}\right\}$ is uniquely determined by $f$. We call it the $\operatorname{domain}$, $\operatorname{Dom} f$, of $f$, and if $f=1$, we define Dom $f=\{1\}$. We define the carrier of $f$ to be $\operatorname{Car} f=\operatorname{Max} \operatorname{Dom} f$.

We say that two elements $e$ and $f$ of $F^{1}$ are $\sim$ equivalent if they have the same carrier: $e \sim f$ if and only if Car $e=\operatorname{Car} f$.

LeMMA 2. $\sim$ is a congruence on $F^{1}$.

Proof. $\sim$ is clearly an equivalence relation; that it is a congruence follows from the fact that $\leqq$ is a partial order on $G$.

We shall denote $F^{1} / \sim$ by $E^{1}$. Note that 1 is the sole element of its $\sim$ equivalence class. Set $E=E^{1} \mid\{1\}$. Effectively also $E=F / \sim$.

It will frequently be convenient to denote any element $f \sim$ of $E^{1}$ simply by $f$. We shall consequently allow ourselves when following this convention to replace $f$ at any stage by any word of $F^{1} \sim$ equivalent to it.

LEMMA 3. $E$ is a semilattice and $E^{\mathfrak{1}}$ is a semilattice obtained from $E$ by the adjunction of an identity element 1.

Relative to the natural order of the semilattice, $e \leqq f$ if and only if Car $f$ $\leqq$ Car $e$, for $e, f \in E^{1}$. Hence the set of maximal elements relative to its natural order, of $E$, is

$$
\left\{x x^{-1} \mid x \in X\right\} \cup\left\{x^{-1} x \mid x \in X\right\} .
$$

Proof. Clearly, if $e, f \in E^{1}$, then

$$
\operatorname{Car} e^{2}=\operatorname{Car} e \text {, }
$$

and

Thus $E^{1}$ is a semilattice.

$$
\text { Car } e f=\text { Car } f e \text {. }
$$

Now $e \leqq f$ in the semilattice $E^{1}$, relative to its natural order, means that $e f=e$. But $e f=e$, i.e. ef $\sim e$, if and only if $\operatorname{Car}(e f)=\operatorname{Car} e$. Since $\operatorname{Car}(e f)$ $=\operatorname{Max}(\operatorname{Car} e \cup \operatorname{Car} f)$, therefore $\operatorname{Car}(e f)=\operatorname{Car} e$ if and only if $\operatorname{Car} f \leqq \operatorname{Car} e$. 
Each element $x$ of $G$ determines a transformation $\varepsilon_{x}$ of $E^{1}$ defined as follows. If $e=1$, we set $e \varepsilon_{x}=x x^{-1}$. If $e=g_{1} g_{1}^{-1} \cdots g_{k} g_{k}^{-1}$ then we set

$$
e \varepsilon_{x}=x x^{-1}\left(x \cdot g_{1}\right)\left(x \cdot g_{1}\right)^{-1} \cdots\left(x \cdot g_{k}\right)\left(x \cdot g_{k}\right)^{-1} \text {. }
$$

We must show that the $\varepsilon_{x}$ are well-defined on $E^{1}$, i.e. we must show that, if $e \sim f$, then $e \varepsilon_{x} \sim f \varepsilon_{x}$. The following lemma provides what is needed. If $A \subseteq G$ and $x \in G$, set $x \cdot A=\{x \cdot a \mid a \in A\}$.

LEMMA 4. Let $A \subseteq G$ and $x \in G$. Let $a \in A$ and suppose that $x \cdot a$ is a maximal element of $x \cdot A$. Then either $a$ is a maximal element of $A$ or $x \cdot a \leqq x$.

Proof. Suppose that $a$ is not a maximal element of $A$; then there exists $b$ in $A$ such that $a<b$, i.e. such that $b=a c=a \cdot c$ for some $c \neq 1$. This implies that $x \cdot a<x \cdot b$ unless the process of reducing the words absorbs the whole of $a$, i.e. unless $x=x^{\prime} a^{-1}=x^{\prime} \cdot a^{-1}$ for some $x^{\prime}$. (In such a case $x \cdot a=x^{\prime}$, while $x b$ $=x^{\prime} c$, and any further reduction between $x^{\prime}$ and $c$ will lead to a situation where $x \cdot a<x \cdot b$.) But if $x=x^{\prime} \cdot a^{-1}$ then

$$
x \cdot a=x^{\prime} \leqq x .
$$

Now suppose that $e \sim f, e, f \in F^{1}$. If $e=1$, then $f=1$ and so $e \varepsilon_{x}=f \varepsilon_{x}$. If $e=g_{1} g_{1}^{-1} \cdots g_{k} g_{k}^{-1}$, set $A=\operatorname{Dom} e$. Then Car $e \varepsilon_{x}=\operatorname{Max}(\{x\} \cup x \cdot A)$, the possibility that some of the $x \cdot g_{i}$ equal 1 not affecting this statement. From Lemma 4 , if $x \cdot a . a \in A$, is a maximal element of $x \cdot A$, either $a$ is a maximal element of $A$, i.e. $a \in \operatorname{Car} e$, or $x \cdot a \leqq x$. Thus $\operatorname{Car} e \varepsilon_{x}=\operatorname{Max}(\{x\} \cup x \cdot \operatorname{Car} e)$. Since Car e $=\operatorname{Car} f$, therefore $\operatorname{Car} e \varepsilon_{x}=\operatorname{Car} f \varepsilon_{x}$, i.e. $e \varepsilon_{x} \sim f \varepsilon_{x}$.

We shall frequently write $e^{x}$ for $e \varepsilon_{x}$.

LEMMA 5. For each $x$ in $G, \varepsilon_{x}$ is an endomorphism of $E^{1}$.

ProOF. Let $e, f \in F^{1}$. If $e=1$, then $(e f)^{x}=f^{x}$ and $e^{x} f^{x}=x x^{-1} f^{x} \sim f^{x}$ from the definition of $\sim$. Similarly, if $f=1,(e f)^{x}=e^{x} f^{x}$.

Suppose that $e \neq 1$ and $f \neq 1$. Set $A=\operatorname{Dom} e$ and $B=\operatorname{Dom} f$. Then $\operatorname{Dom}(e f)$ $=A \cup B$. Hence

$$
\begin{aligned}
\operatorname{Car}(e f)^{x} & =\operatorname{Max}(\{x\} \cup x \cdot(A \cup B)) \\
& =\operatorname{Max}((\{x\} \cup x \cdot A) \cup(\{x\} \cup x \cdot B)) \\
& =\operatorname{Car} e^{x} f^{x} .
\end{aligned}
$$

Thus $(e f)^{x} \sim e^{x} f^{x}$. This completes the proof.

The mapping $x \rightarrow \varepsilon_{i}, x \in G$, is almost an antihomomorphism of $G$ into End $E^{1}$. The extent to which it fails to be is clarified in the next lemma. 
Lemma 6. Let $e \in E^{1}, x, y \in G$. Then $x x^{-1} e^{x_{*} y}=\left(e^{y}\right)^{x}$.

Proof. If $e=1$, then

$$
\begin{aligned}
\left(e^{y}\right)^{x} & =\left(y y^{-1}\right)^{x} \\
& = \begin{cases}x x^{-1}(x \cdot y)(x \cdot y)^{-1}, & \text { if } y \neq 1 \\
x x^{-1} & , \text { if } y=1\end{cases} \\
& \sim x x^{-1}(x \cdot y)(x \cdot y)^{-1}, \text { in both cases, } \\
& =x x^{-1} e^{x \cdot y} .
\end{aligned}
$$

If $e=g_{1} g_{1}^{-1} \cdots g_{k} g_{k}^{-1}$, then

$$
\left(e^{y}\right)^{x}=\left(y y^{-1}\left(y \cdot g_{1}\right)\left(y \cdot g_{1}\right)^{-1} \cdots\left(y \cdot g_{k}\right)\left(y \cdot g_{k}\right)^{-1}\right)^{x} .
$$

If $y \neq 1$ and also no $y \cdot g_{i}$ equals 1 , then

$$
\begin{aligned}
\left(e^{y}\right)^{x} & =x x^{-1}(x \cdot y)(x \cdot y)^{-1}\left(x \cdot y \cdot g_{1}\right)\left(x \cdot y \cdot g_{1}\right)^{-1} \cdots\left(x \cdot y \cdot g_{k}\right)\left(x \cdot y \cdot g_{k}\right)^{-1} \\
& =x x^{-1} e^{x \cdot y}
\end{aligned}
$$

as required. If $y=1$, then

by definition of $\sim$,

$$
\left(e^{y}\right)^{x}=e^{x}=x x^{-1} e^{x}
$$

$$
=x x^{-1} e^{x, y}
$$

as required.

Finally, suppose that $y \neq 1$, and that some $y \cdot g_{i}$ equal 1 . Then the corresponding factors $\left(y \cdot g_{i}\right)\left(y \cdot g_{i}\right)^{-1}$ will be suppressed in the right-hand side of (1) above. Hence, in evaluating this right-hand side the corresponding expressions $\left(x . y . g_{i}\right)\left(x \cdot y \cdot g_{i}\right)^{-1}$ will not occur. However, since each such expression is equal to $x x^{-1}$, and $x x^{-1}$ already occurs on the right-hand side, from the definition of $\sim$, we have, with no factors missing, from (1),

$$
\begin{aligned}
& \left(e^{y}\right)^{x}=x x^{-1}(x \cdot y)(x \cdot y)^{-1}\left(x \cdot y \cdot g_{1}\right)\left(x \cdot y \cdot g_{1}\right)^{-1} \cdots\left(x \cdot y \cdot g_{k}\right)\left(x \cdot y \cdot g_{k}\right)^{-1} \\
& =x x^{-1} e^{x . y}
\end{aligned}
$$

completing the proof.

We can now define, in terms of the endomorphisms $\varepsilon_{x}$, the semi-direct product $J_{X}^{1}$ as the set $E^{1} \times G$ on which a product is given by

$$
(e, x)(f, y)=\left(e f^{x}, x \cdot y\right) .
$$

LEMMA 7. $J_{X}^{1}$ is a semigroup.

Proof. Let $(e, x),(f, y),(g, z) \in E^{1} \times G$. Then 


$$
\begin{aligned}
((e, x)(f, y))(g, z) & =\left(e f^{x}, x \cdot y\right)(g, z)=\left(e f^{x} g^{x \cdot y},(x \cdot y) \cdot z\right) \\
& =\left(e f^{x} x x^{-1} g^{x, y}, x \cdot(y \cdot z)\right),
\end{aligned}
$$

by the definition of $\sim$, since $f^{x} \sim x x^{-1} f^{x}$,

by Lemma 6 ,

$$
=\left(e f^{x}\left(g^{y}\right)^{x}, x(y \cdot z)\right)
$$

since $\varepsilon_{x} \in$ End $E^{1}$,

$$
\begin{aligned}
& =(e, x)\left(f g^{y}, y \cdot z\right), \\
& =(e, x)((f, y)(g, z)) .
\end{aligned}
$$

We now find the idempotents and the regular elements of $J_{X}$.

LEMMA 8. $E^{1} \times\{1\}$ is the set of idempotents of $J_{X}^{1}$. It forms a subsemigroup of $J_{X}^{1}$ isomorphic to $E^{1}$.

Proof. This result is immediate from the definition of the product in $J_{X}^{1}$. For $(e, x)^{2}=(e, x)$ if and only if $x \cdot x=x$, i,e. if and only if $x=1$.

Lemma 9. Let $(e, x) \in J_{X}^{1}$. Then $(e, x)$ is a regular element of $J_{X}^{1}$ if and only if $\{x\} \leqq \operatorname{Car} e$, i.e. if and only if Car $x x^{-1} e=\operatorname{Car} e$.

Proof. Let $(e, x)$ be regular. Then there exists $(f, y)$ such that $(e, x)(f, y)(e, x)$ $=(e, x)$. Hence

$$
\left(e f^{x} e^{x, y}, x \cdot y \cdot x\right)=(e, x) .
$$

Thus $x \cdot y \cdot x=x$, so that $x \cdot y=1$, and

i.e.

$$
e f^{x} e^{1}=e
$$

$$
e x x^{-1} f^{x} e=e
$$

From the definition of $\sim$ it follows that $\{x\} \leqq$ Car $e$.

Conversely, suppose that $\{x\} \leqq$ Car $e$. Set $y=x^{-1}$; then

$$
(e, x)\left(e^{y}, y\right)(e, x)=\left(e\left(e^{y}\right)^{x} e, x\right) .
$$

By Lemma $6,\left(e^{y}\right)^{x}=x x^{-1} e^{x \cdot y}=x x^{-1} e^{1}=x x^{-1} e$. However, since $\{x\} \leqq$ Car $e$, Car $x x^{-1} e=$ Car $e$. Hence $x x^{-1} e=e$. Thus $e x x^{-1} e e=e$ and

$$
(e, x)\left(e^{y}, y\right)(e, x)=(e, x) \text {. }
$$

We may now proceed in two ways. The first way is that of the next lemma.

LEMMA 10. The regular elements of $J_{X}^{1}$ form an inverse subsemigroup of $J_{X}^{1}$.

Proof. Let $a, b$ be regular elements of $J_{X}^{1}$. Let $a^{\prime}, b^{\prime}$ be inverses of $a, b$, respectively. Then, since the idempotents of $J_{X}^{1}$ commute, by Lemmas 3 and 8 , 
and similarly,

$$
a b\left(b^{\prime} a^{\prime}\right) a b=a\left(b b^{\prime}\right)\left(a^{\prime} a\right) b=\left(a a^{\prime} a\right)\left(b b^{\prime} b\right)=a b,
$$

Thus $a b$ is regular.

$$
b^{\prime} a^{\prime}(a b) b^{\prime} a^{\prime}=b^{\prime} a^{\prime} .
$$

Since the idempotents of this regular subsemigroup form a semilattice it forms an inverse semigroup.

COROLlARY. If $(e, x)$ is a regular element of $J_{X}^{1}$ then it has a unique inverse, namely $\left(e^{x^{-1}}, x^{-1}\right)$, in $J_{x}^{1}$.

Proof. That any inverse is unique follows from Lemma 10 because any inverse of a regular element is itself regular. That $\left(e^{x^{-1}}, x^{-1}\right)$ is the inverse of $(e, x)$ when it is regular was part of the proof of Lemma 9.

Let us denote the semigroup of regular elements of $J_{X}^{1}$, i.e., by Lemma 10 , the maximal inverse subsemigroup of $J_{X}^{1}$, by $I_{X}^{1}$.

The second possible construction of $I_{X}^{1}$ is as a homomorphic image of $J_{X}^{1}$. We shall say that $(e, x) \sim(f, y)$ if and only if $x=y$ and Car $x x^{-1} e=\operatorname{Car} y y^{-1}$ It is immediate that $(e, 1) \sim(f, 1)$ if and only if $e \sim f$, where the latter $\sim$ denotes our earlier equivalence on $E^{1}$. Since we have already seen (Lemma 8) that $E^{1} \times\{1\}$ may be identified with $E^{1}$, our new definition of $\sim$ may be regarded as merely extending $\sim$ from $E^{1}$ to $J_{X}^{1}$.

LEMMA 11. $\sim$ is a congruence on $J_{X}^{1}$.

Proof. Let $(e, x),(f, y),(g, z) \in J_{X}^{1}$ and suppose that $(e, x) \sim(f, y)$. Thus $x=y$ and $\operatorname{Car} x x^{-1} e=\operatorname{Car} y y^{-1} f$, i.e. Car $x x^{-1} e=\operatorname{Car} y y^{-1} f$.

To prove right compatibility, consider

and

$$
(e, x)(g, z)=\left(e g^{x}, x \cdot z\right)
$$

$$
(f, y)(g, z)=\left(f g^{x}, x \cdot z\right),
$$

since $x=y$. Now, by the definition of $\sim$ on $F^{1}, g^{x} \sim x x^{-1} g^{x}$, so by Lemma 2,

$$
\begin{aligned}
(x \cdot z)(x \cdot z)^{-1} e g^{x} & \sim(x \cdot z)(x \cdot z)^{-1} \epsilon x x^{-1} g^{x} \\
& \sim(x \cdot z)(x \cdot z)^{-1} f x x^{-1} g^{x},
\end{aligned}
$$

since $e x x^{-1} \sim f x x^{-1}$

$$
\sim(x \cdot z)(x \cdot z)^{-1} f g^{x}
$$

hence

$$
(e, x)(g, z) \sim(f, y)(g, z) .
$$

To deal with left compatibility, consider

and

$$
(g, z)(e, x)=\left(g e^{z}, z \cdot x\right)
$$


since $x=y$. Since

$$
(g, z)(f, y)=\left(g f^{z}, z \cdot x\right),
$$

$$
x x^{-1} e \sim x x^{-1} f,
$$

therefore,

$$
\left(x x^{-1} e\right) \varepsilon_{z} \sim\left(x x^{-1} f\right) \varepsilon_{z},
$$

so, by Lemma 5 ,

$$
z z^{-1}(z \cdot x)(z \cdot x)^{-1} e^{z} \sim z z^{-1}(z \cdot x)(z \cdot x)^{-1} f^{z} .
$$

Thus

$$
(z \cdot x)(z \cdot x)^{-1} e^{z} \sim(z \cdot x)(z \cdot x)^{-1} f^{z} .
$$

By Lemma 2, therefore

$$
(z \cdot x)(z \cdot x)^{-1} g e^{z} \sim(z \cdot x)(z \cdot x)^{-1} g f^{z} .
$$

Hence $\sim$ is right compatible.

LEMMA 12. Each congruence class of $\sim$ in $J_{X}^{1}$ contains precisely one regular element. Hence $J_{X}^{1} l \sim \cong I_{X}^{1}$.

Proof. The final statement follows immediately from Lemma 10, for the set of regular elements of $J_{X}^{1}$ form the inverse semigroup $I_{X}^{1}$.

Let $(e, x) \in J_{X}^{1}$. Then

$$
\left(x x^{-1} e, x\right) \sim(e, x)
$$

and, by Lemma $9,\left(x x^{-1} e, x\right)$ is regular. Thus each $\sim$ class contains a regular element.

Suppose that $(e, x) \sim(f, y)$ and that $(e, x)$ and $(f, y)$ are regular. Since $(e, x)$ $\sim(f, y)$, therefore $x=y$ and $\operatorname{Car}\left(x x^{-1} e\right)=\operatorname{Car}\left(y y^{-1} f\right)$. Since $(e, x)$ is regular, Car $x x^{-1} e=$ Car $e$; since $(f, y)$ is regular, Car $\left(y y^{-1} f\right)=\operatorname{Car} f$. Thus $x=y$ and Car $e=\operatorname{Car} f$, i.e. $e \sim f$. Thus $(e, x)=(f, y)$.

We now define $I_{X}$ to be $I_{X}^{1} \backslash\{1\} . I_{X}$ is clearly an inverse subsemigroup of $I_{X}^{1}$. Define $\phi: X \rightarrow I_{X}$ thus

$$
\phi: x \rightarrow\left(x x^{-1}, x\right), x \in X .
$$

Then $\phi$ is one-to-one i.e. $\phi$ embeds $X$ in $I_{X}$. In the next section we show that $\left(I_{X}, \phi\right)$ is a free inverse semigroup on $X$.

We comment now an another possible construction of $I_{X}$, mentioned earlier, starting from the set $W$. We define an equivalence $\sim$, say, on $W$ thus:

$$
f g \sim e h \text { if and only if } g=h \text { and } \operatorname{Car} f g g^{-1}=\operatorname{Car} e h h^{-1},
$$

for $f, e \in F^{1}, g, h \in G, f g, e h \in W$. On $W / \sim$ we then define a product as follows:

$$
((f g) \sim)((e h) \sim)=\left(f g g^{-1} k g \cdot h\right) \sim,
$$

where, if $e=h_{1} h_{1}^{-1} \cdots h_{i} h_{l}^{-1}$, then 


$$
k=\left(g \cdot h_{1}\right)\left(g \cdot h_{1}\right)^{-1} \cdots\left(g \cdot h_{l}\right)\left(g \cdot h_{l}\right)^{-1} .
$$

With this product we then take $W / \sim$ as $I_{X}$.

\section{The freedom of $\left(I_{X}, \phi\right)$}

In what follows we take $I_{X}$ as the subsemigroup of $J_{X}^{1}$ consisting of all the regular elements of $J_{X}^{1}$ other than 1 .

We are to show that $\left(I_{X}, \phi\right)$ is a free inverse semigroup on $X$. In other words, we are to show that if $S$ is an inverse semigroup and $\alpha: X \rightarrow S$ is any mapping, then there is a unique morphism $\theta: I_{X} \rightarrow S$, say, such that $\phi \theta=\alpha$.

To construct $\theta$, first let us adjoin an identity element 1 , an additiona one if $S$ already has one, to $S$ to form the inverse semigroup $S^{*}$. Define $\beta: G \rightarrow S^{*}$ as follows:

$$
\begin{aligned}
x \beta & =x \alpha, x \in X, \\
x^{-1} \beta & =(x \alpha)^{-1}, x^{-1} \in X^{-1},
\end{aligned}
$$

where $(x \alpha)^{-1}$ is the inverse in $S^{*}$,

$$
\begin{aligned}
1 \beta & =1, \\
\left(y_{1} y_{2} \cdots y_{n}\right) \beta & =\left(y_{1} \beta\right)\left(y_{2} \beta\right) \cdots\left(y_{n} \beta\right), y_{i} \in Y,
\end{aligned}
$$

where $y_{1} y_{2} \cdots y_{n}$ is reduced.

Lemma 13. Let $g, h \in G$ and suppose that $g \leqq h$. Then

in $S^{*}$.

$$
(g \beta)(g \beta)^{-1} \leqq(h \beta)(h \beta)^{-1}
$$

Proof. There exists $g^{\prime}$ in $G$ such that $h=g g^{\prime}=g \cdot g^{\prime}$. Hence, from the definition of $\beta$,

Thus

$$
h \beta=(g \beta)\left(g^{\prime} \beta\right) .
$$

$$
\begin{aligned}
(g \beta)(g \beta)^{-1}(h \beta)(h \beta)^{-1} & =(g \beta)(g \beta)^{-1}(g \beta)\left(g^{\prime} \beta\right)(h \beta)^{-1} \\
& =(g \beta)\left(g^{\prime} \beta\right)(h \beta)^{-1} \\
& =(h \beta)(h \beta)^{-1} .
\end{aligned}
$$

We may now define $\theta$ as follows. Let $(e, g) \in I_{X}$. Then $e \neq 1$, for Car $g g^{-1} e$ $=$ Car $e$, and $(e, g) \neq(1,1)$. Hénce $e=g_{1} g_{1}^{-1} \cdots g_{k} g_{k}^{-1}$, say. Define

$$
(e, g) \theta=\left(g_{1} \beta\right)\left(g_{1} \beta\right)^{-1} \cdots\left(g_{k} \beta\right)\left(g_{k} \beta\right)^{-1} g \beta
$$

where, on the right of this equation, $\left(g_{i} \beta\right)^{-1}, i=1, \cdots, k$, denotes the inverse of $g_{i} \beta$ in $S$. From Lemma 13, if $e \sim f$ (in $F^{1}$ ), so that $(e, g)=(f, g)$ in $I_{X}$, then the above definition gives $(e, g) \theta=(f, g) \theta$. Thus $\theta$ is well-defined. 
The following lemma contains two results needed to show that $\theta$ is a morphism.

Lemma 14. Let $T$ be any inverse semigroup. Let $a, b, m \in T$. Then

$$
\begin{aligned}
(a m)\left(m^{-1} b\right)\left(m^{-1} b\right)^{-1} & =(a b)(a b)^{-1} a m, \\
(a m)\left(m^{-1} b\right) & =(a m)(a m)^{-1} a b .
\end{aligned}
$$

PROOF. (i) $(a m)\left(m^{-1} b\right)\left(m^{-1} b\right)^{-1}=a \cdot m m^{-1} \cdot b b^{-1} \cdot m=a b b^{-1} m m^{-1} m$

$$
=a \cdot a^{-1} a \cdot b b^{-1} \cdot m=a b b^{-1} a^{-1} a m .
$$

$$
(a m)\left(m^{-1} b\right)=a \cdot a^{-1} a \cdot m m^{-1} \cdot b=a m m^{-1} a^{-1} \cdot a b .
$$

Consider now $(e, g),(f, h)$ in $I_{X}$. Then

$$
(e, g)(f, h)=\left(e f^{g}, g \cdot h\right) \text {. }
$$

Since $e$ and $f$ are not equal to 1 , we have $e=g_{1} g_{1}^{-1} \cdots g_{k} g_{k}^{-1}$ and $f=h_{1} h_{1}^{-1}$ $\cdots h_{l} h_{l}^{-1}$, say. Set $g_{i} \beta=s_{i}, i=1, \cdots, k, h_{j} \beta=t_{j}, j=1,2, \cdots, l, g \beta=s$, and $h \beta$ $=t$.

Suppose that $g \cdot h=g^{\prime} u \cdot u^{-1} h^{\prime}$, where $g=g^{\prime} u, h=u^{-1} h^{\prime}$, and $g \cdot h$ $=g^{\prime} \cdot h^{\prime} h g=g^{\prime} h^{\prime}$, and that

where

$$
g \cdot h_{j}=g^{(j)} u_{j} \cdot u_{j}^{-1} h_{j}^{\prime}
$$

$$
g^{(j)} u_{j}=g, u_{j}^{-1} h_{j}^{\prime}=h_{j}, g \cdot h_{j}=g^{(j)} \cdot h_{j}^{\prime}=g^{(j)} h_{j}^{\prime},
$$

for $j=1, \cdots, l$. Set $g^{(j)} \beta=s^{(j)}, u_{j} \beta=r_{j}, h_{j}^{\prime} \beta=t_{j}^{\prime}, g^{\prime} \beta=s^{\prime}, h^{\prime} \beta=t^{\prime}$, and $u \beta=r$.

Then, by definition of $\theta$,

$$
\begin{aligned}
\left(e f^{g}, g \cdot h\right) \theta & =\left(g_{1} g_{1}^{-1} \cdots g_{k} g_{k}^{-1} g g^{-1}\left(g \cdot h_{1}\right)\left(g \cdot h_{1}\right)^{-1} \cdots\left(g \cdot h_{l}\right)\left(g \cdot h_{l}\right)^{-1}, g \cdot h\right) \theta \\
& =s_{1} s_{1}^{-1} \cdots s_{k} s_{k}^{-1} s s^{-1}\left(g \cdot h_{1}\right) \beta\left(\left(g \cdot h_{1}\right) \beta\right)^{-1} \cdots\left(g \cdot h_{l}\right) \beta\left(\left(g \cdot h_{l}\right) \beta\right)^{-1}(g \cdot h) \beta \\
& =s_{1} s_{1}^{-1} \cdots s_{k} s_{k}^{-1} s s^{-1}\left(s^{(1)} t_{1}^{\prime}\right)\left(s^{(1)} t_{1}^{\prime}\right)^{-1} \cdots\left(s^{(l)} t^{\prime}\right)\left(s^{(l)} t^{\prime}\right)^{-1} s^{\prime} t^{\prime} .
\end{aligned}
$$

On the other hand,

$$
\begin{aligned}
(e, g) \theta(t, h) \theta & =\left(s_{1} s_{1}^{-1} \cdots s_{k} s_{k}^{-1} s\right)\left(t_{1} t_{1}^{-1} \cdots t_{l} t_{l}^{-1} t\right) \\
& =s_{1} s_{1}^{-1} \cdots s_{k} s_{k}^{-1} s s^{-1}\left(s t_{1} t_{1}^{-1}\right) \cdots t_{l} t_{l}^{-1} t \\
& \left.=s_{1} s_{1}^{-1} \cdots s_{k} s_{k}^{-1} s s^{-1}\left(s^{(1)} t_{1}\right)\left(s^{(1)} t_{1}\right)^{-1}\right) s t_{2} t_{2}^{-1} \cdots t_{l} t_{l}{ }^{-1} t \\
& =s_{1} s_{1}^{-1} \cdots s_{k} s_{k}^{-1} s s^{-1}\left(s^{(1)} t_{1}^{\prime}\right)\left(s^{(1)} t_{1}^{\prime}\right)^{-1} \cdots\left(s^{(l)} t_{l}^{\prime}\right)\left(s^{(l)} t_{l}^{\prime}\right)^{-1} s t
\end{aligned}
$$

by successive applications of Lemma 14, part (i),

$$
=s_{1} s_{1}^{-1} \cdots s_{k} s_{k}^{-1} s s^{-1}\left(s^{(1)} t_{1}^{\prime}\right)\left(s^{(1)} t_{1}^{\prime}\right)^{-1} \cdots\left(s^{(l)} t^{\prime}\right)\left(s^{(l)} t_{i}^{\prime}\right)^{-1} s s^{-1} s^{\prime} t^{\prime},
$$


by Lemma 14, part (ii),

$$
=\left(e f^{g}, g \cdot h\right) 9,
$$

completing the proof that $\theta$ is a morphism.

Now, immediately from the definition of $\theta$,

$$
\begin{aligned}
x \phi \theta & =\left(x x^{-1}, x\right) \theta \\
& =(x \alpha)(x \alpha)^{-1}(x \alpha) \\
& =x \alpha, \text { if } x \in X .
\end{aligned}
$$

Thus $\phi \theta=\alpha$. It remains to show that $\theta$ is uniquely determined by this condition. This will be so if $X \phi$ is a set of generators of $I_{X}$. This is so, for we easily check that, if $e=g_{1} g_{1}^{-1} \cdots g_{k} g_{k}^{-1}$ then

$$
(e, g)=\left(g_{1} g_{1}^{-1}, 1\right) \cdots\left(g_{k} g_{k}^{-1}, 1\right)\left(g g^{-1}, g\right),
$$

and for any $h$ in $G$,

$$
\left(h h^{-1}, 1\right)=\left(h h^{-1}, h\right)\left(h^{-1} h, h^{-1}\right) .
$$

Moreover, if $h=y_{1} \cdots y_{n}, y_{i} \in Y$, then

$$
\left(h h^{-1}, h\right)=\left(y_{1} y_{1}^{-1}, y_{1}\right)\left(y_{2} y_{2}^{-1}, y_{2}\right) \cdots\left(y_{n} y_{n}^{-1}, y_{n}\right),
$$

a product of elements of $X \phi$ and their inverses.

The final remarks of the above proof lead to a canonical form for the elements of $I_{X}$. If $(e, g) \in I_{X}$, we may choose $e$, by the definition of $\sim$ (on $F^{1}$ ), so that Car $e$ $=$ Dom $e$. Suppose that then $e=g_{1} g_{1}^{-1} \cdots g_{k} g_{k}^{-1}$. Then $e$ is uniquely expressible in this form, modulo $\sim$, up to a permutation of the factors $g_{i} g_{i}^{-1}$. Modulo such permutations, equations (1), (2) and (3), above, enable each element of $I_{X}$ to be expressed as a unique product of elements of $X$ and their inverses.

\section{References}

[1] H. E. Scheiliblich, 'Free Inverse Semigroups', Semigroup Forum 4 (1972), 351-359.

[2] W. D. Munn, 'Fundamental Inverse Semigroups', Quart. J. Math. Oxford Ser. (2) 21 (1970), 157-170.

Monash University

Clayton, Victoria, 3168

Australia. 